\title{
Mechanical and metabolic profile of locomotion in adults with childhood-onset GH deficiency
}

\author{
Alberto E Minetti ${ }^{1,2,3}$, Luca P Ardigò ${ }^{2}$, Franco Saibene ${ }^{3}$, Simona Ferrero ${ }^{4}$ and Alessandro Sartorio ${ }^{4,5}$ \\ ${ }^{1}$ Exercise and Sport Science, Manchester Metropolitan University, Alsager, UK, ${ }^{2}$ CSAM-Ergonomia, Fondazione S Maugeri, IRCCS, Pavia, Italy, \\ ${ }^{3}$ Reparto di Fisiologia, ITBA, CNR, Segrate (MI), Italy, ${ }^{4}$ Centro per i Disordini della Crescita, LSRE, Istituto Auxologico Italiano, IRCCS, Milan, Italy, \\ and ${ }^{5}$ Divisione Malattie Metaboliche, Istituto Auxologico Italiano, IRCCS, Piancavallo (VB), Italy
}

(Correspondence should be addressed to A Sartorio, Laboratorio Sperimentale di Ricerche Endocrinologiche (LSRE), Istituto Auxologico Italiano, IRCCS, Via Ariosto 13-20145 Milan, Italy; Email: sartorio@auxologico.it)

\begin{abstract}
Objective: The aim of the present study was to evaluate the energy cost and the mechanical work of locomotion in a group of adults with childhood-onset GH deficiency (GHD).

Subjects: Eight males with childhood-onset GHD (mean age \pm s.D.: $31.7 \pm 3.6$ years; mean height: $145.1 \pm 6.7 \mathrm{~cm}$ ) and six age-, sex- and exercise-matched normal subjects were studied.

Design: GHD patients and healthy controls were requested to walk and run in the speed range of $2-11 \mathrm{~km} \mathrm{~h}^{-1}$. For each condition, simultaneous mechanical and metabolic measurements were taken. Methods: Oxygen consumption, and mechanical internal and external work of locomotion were evaluated with standard open-circuit respirometry and three-dimensional motion analysis respectively. Results: External work was not significantly different between GHD patients and healthy controls, while internal work was higher for patients at all speeds. In walking, the relationships between both the mechanical energy recovery and the metabolic cost with speed were shifted towards lower speeds in patients. As a consequence, the optimal speed of walking, i.e. the speed at which the cost of locomotion is minimum, was lower for GHD patients. Stride frequency was significantly higher $(11.2-11.3 \%)$ for GHD patients at all speeds of walking and running. GHD patients were unable to run at speeds higher than $8 \mathrm{~km} \mathrm{~h}^{-1}$ for the time needed to reach a metabolic steady state.

Conclusion: It appears that both the mechanics and energetics of locomotion in short-statured adults with childhood-onset GHD are not strikingly different from those of healthy controls, thus demonstrating a substantial 'normality' in this group of GHD patients at metabolically attainable speeds. The 'harmonic' body structure and the adherence to allometric transformations in these patients do not exclude the possibility of a different metabolic role of GH in normally statured adults with childhood-onset GHD and in those with acquired GHD, taking into account the well recognized heterogeneity of the adult GHD syndrome.
\end{abstract}

European Journal of Endocrinology 142 35-41

\section{Introduction}

Several studies reported that growth hormone $(\mathrm{GH})$ deficiency (GHD) in adults is associated with reduced maximal isometric muscle strength, muscle size and muscle fibre area $(1-6)$, thereby indicating the importance of $\mathrm{GH}$ in adults for the maintenance of muscle mass and strength.

The majority of clinical studies, recruiting 'mixed' study groups (childhood- and adult-onset GHD), used 'absolute' values of strength and power for comparison between controls and patients. Although this choice can be considered acceptable in patients with adultonset GHD who usually have normal body dimensions (their height, weight and body mass index (BMI) are perfectly matched with those of healthy controls), it appears highly questionable in short-statured adults with childhood-onset GHD.
In our experience, once actual quadriceps and handgrip strengths were normalized for cross sectional area (CSA), no differences were found between adults with childhood-onset GHD and controls $(3,5)$, thus suggesting that reduced muscular size and strength are probably related to a simple dimensional down-scaling rather than to a real impairment of muscle function.

Taking into account this statement, differences between childhood- and adult-onset GHD syndrome might be more evident than previously believed; thus, separate data analysis between patients with very different clinical histories and duration of lack of $\mathrm{GH}$-insulin-like growth factor (IGF)-I seems essential.

Adults with adult-onset GHD, having normally developed body size with reduced muscle mass and strength, might undergo more dramatic consequences of muscle mass decrease than adults with childhoodonset GHD who, lacking GH during the period of 
maximal growth, have a proportional decrease in body size, muscle mass and strength (7).

The different age at which GHD sets in might also influence the proportion of fibre types in a different way. In this respect, Rutherford et al. (4) provided some indirect evidence, based on lower half-relaxation time and rightward shift of force-frequency in relation to quadriceps muscle, supporting the presence of a higher proportion of fast twitch, type 2 muscle fibres in adult-onset GHD patients. However, in a recent study where direct measurements were performed on vastus lateralis biopsy samples, we demonstrated that fibre type distribution (i.e. myosin heavy chain isoform composition) and type 1 and $2 \mathrm{~A}$ muscle fibre CSA/height ${ }^{2}$ in adults with childhood-onset GHD are not different from controls (7).

To our knowledge, no attempt has been made so far to evaluate the influence of chronic lack of GH-IGF-I on those activities more related to daily life habits, such as ordinary locomotion (walking and running), in adults with GHD. For this reason, the aim of the present study was to evaluate the energy cost and the mechanical work of locomotion in a very strictly selected, although necessarily limited, group of patients with childhood-onset GHD.

\section{Materials and methods}

Eight males with childhood-onset GHD were admitted to the study. The main clinical data of patients are shown in Table 1.

All patients had received GH substitution therapy during childhood; previous GH therapy had been interrupted at least 7 years before entry into the present study.

The diagnosis of GHD (GH peak $<5 \mathrm{ng} / \mathrm{ml}$ ) was confirmed in each subject by means of two GH stimulation tests (insulin-induced hypoglycaemia, L-dopa plus propranolol and/or GH releasing hormone (GHRH)); mean plasma IGF-I levels of patients (mean \pm S.D.: $54.1 \pm$ $17.1 \mu \mathrm{g} / \mathrm{l})$ were significantly lower $(P<0.001)$ than those recorded in normal adults $(296.3 \pm 66.2 \mu \mathrm{g} / \mathrm{l}$; range, $174.0-397.2 \mu \mathrm{g} / \mathrm{l})$.

Two patients (nos 1 and 5) had, in addition, associated hormone deficiencies and therefore they received adequate stable substitution therapy; no relevant modification in the dosages of hormonal replacement treatment had been necessary in the last 3 years.

The data obtained were compared with those recorded in a healthy control group $(n=6)$, matched for sex, age and exercise, but different for height (176.0 \pm $4.3 \mathrm{~cm})$, recruited among friends and colleagues.

\section{Hormone determinations}

Serum GH and plasma IGF-I levels were determined using commercial RIA kits (Sorin, Saluggia, Italy and Nichols, San Juan Capistrano, USA respectively); the intra- and interassay coefficients of variation were less than $5 \%$.

\section{Study protocol}

The nature of the trial was explained to the patients and their written informed consent was obtained. The study protocol was approved by the local Ethical Committee.

GHD patients and healthy controls, after $10-15 \mathrm{~min}$ habituation on a motor-driven treadmill, were requested to walk in the speed range of $2-8$ and $3-9 \mathrm{~km} \mathrm{~h}^{-1}$ (step $1 \mathrm{~km} \mathrm{~h}^{-1}$ ) respectively, and to run in the speed range of 6-11 and 7-11 $\mathrm{km} \mathrm{h}^{-1}$ on a motor-driven treadmill. For each condition, simultaneous mechanical and metabolic measurements were taken in 4 patients (nos 1-4); only mechanical measurements were recorded in the other 4 patients (nos 5-8).

Experiments were always carried out in the afternoon, about two hours after the last meal.

\section{Biomechanical measurements}

Each trial was recorded by means of an opto-electronic motion analyser (ELITE System, BTS., Corsico, Italy)

Table 1 Main clinical data of adults with childhood-onset GH deficiency.

\begin{tabular}{|c|c|c|c|c|c|c|c|c|c|c|}
\hline \multirow[b]{2}{*}{ Patient } & \multirow{2}{*}{$\begin{array}{c}\text { Age } \\
\text { (years) }\end{array}$} & \multirow{2}{*}{$\begin{array}{l}\text { Height } \\
\text { (cm) }\end{array}$} & \multirow{2}{*}{$\begin{array}{l}\text { Weight } \\
\text { (kg) }\end{array}$} & \multirow{2}{*}{$\begin{array}{c}\text { BMI } \\
\left(\mathrm{kg} / \mathrm{m}^{2}\right)\end{array}$} & \multirow{2}{*}{$\begin{array}{c}\text { pre GH tx } \\
\text { (months) }\end{array}$} & \multirow{2}{*}{$\begin{array}{l}\text { Wash-out GH tx } \\
\text { (years) }\end{array}$} & \multicolumn{3}{|c|}{ Associated defects } & \multirow[b]{2}{*}{ Medication } \\
\hline & & & & & & & TSH & LH-FSH & ACTH & \\
\hline 1 & 36 & 131.5 & 47.3 & 27.3 & 36 & 17 & + & + & + & $\mathrm{Te}, \mathrm{T}, \mathrm{C}$ \\
\hline 2 & 25 & 145.0 & 51.0 & 24.3 & 60 & 7 & - & - & - & - \\
\hline 3 & 34 & 148.5 & 42.0 & 19.1 & 24 & 20 & - & - & - & - \\
\hline 4 & 32 & 153.0 & 69.0 & 29.5 & 44 & 18 & - & - & - & - \\
\hline 5 & 30 & 150.0 & 54.0 & 24.0 & 72 & 8 & - & + & - & $\mathrm{Te}$ \\
\hline 6 & 34 & 143.5 & 50.0 & 24.3 & 46 & 14 & - & - & - & - \\
\hline 7 & 29 & 148.0 & 48.5 & 22.1 & 66 & 12 & - & - & - & - \\
\hline 8 & 34 & 141.0 & 61.2 & 30.7 & 62 & 16 & - & - & - & - \\
\hline Mean & 31.7 & 145.1 & 52.9 & 25.2 & 51.2 & 14.0 & & & & \\
\hline \pm S.D. & 3.6 & 6.7 & 8.5 & 3.8 & 16.4 & 4.7 & & & & \\
\hline
\end{tabular}

TSH, thyrotropin; LH, luteinizing hormone; FSH, follicle stimulating hormone; ACTH, adrenocorticotrophin; Te, testosterone enanthate; $\mathrm{T}$, thyroxine; $\mathrm{C}$, cortisone acetate; tx, therapy. 
with four cameras sampling at a rate of $100 \mathrm{~Hz}$. A dedicated computer for each camera automatically recognizes the shape of reflective markers, illuminated via stroboscopic infrared light. Nine reflective hemispheric spots (diameter $2 \mathrm{~cm}$ ) were placed on each side of the body in order to identify the subject's segments of interest: the head-trunk (ear lobe-iliac crest), thigh (great trochanter-knee joint), shank (knee joint-ankle malleolus), foot (heel-toe), upper arm (shoulder-elbow) and forearm (elbow-wrist). The three-dimensional positions of segment extremities were stereometrically calculated by the apparatus. Each acquisition lasted $5 \mathrm{~s}$ and the stride frequency was calculated by analysing the periodicity of the vertical coordinates and by counting the number of frames for a stride.

Values for segment mass, centre of mass position and radius of gyration were taken from standard tables (8). Mechanical positive internal (Wint) and external (Wext) work were computed using the method of Cavagna and Kaneko (9). Wint accounts for the kinetic energy changes of segments, the movements of which do not affect the position of the overall centre of mass (i.e. symmetrical limbs displacements), and it is based on the König's theorem from mechanics. This theorem states that the total kinetic energy of a multi-link system can be divided into two parts: (a) the kinetic energy of the segments arising from their change of speed with respect to the overall centre of mass, and (b) the kinetic energy of the overall centre of mass. The first term is the mechanical internal work, Wint, while the second is included in the external work, Wext.

The three-dimensional displacement of the body centre of mass was calculated for each frame from the positions of the 12 body segments. Positive Wext was obtained by summing the increments of the total energy (= potential + horizontal kinetic + vertical kinetic) curve of the body centre of mass versus time.

All the data processing and statistics were performed on an Athena 486 computer and an Apple Macintosh Quadra 700 computer. Low-pass filtering of the obtained spatial coordinates was performed using the method of D'Amico and Ferrigno (10), where optimal filter cut-off frequencies are automatically selected. The range of cut-off frequencies was $5.0-9.0 \mathrm{~Hz}$ using this procedure. A custom-built program, created using LabView2/Macintosh (National Instruments, Austin, TX, USA), analysed the three-dimensional data and calculated the biomechanical variables. The procedure for data differentiation, needed to compute marker speed, was the simple $\mathrm{dx} / \mathrm{dt}, \mathrm{dy} / \mathrm{dt}$ and $\mathrm{dz} / \mathrm{dt}$ calculation on the filtered coordinates.

\section{Metabolic measurements}

Standard open-circuit respirometry was used. Standard temperature pressure dry (STPD) ventilation and $\mathrm{O}_{2}$ uptake were measured by an integrated metabolism analyser (Medical Graphics Corporation, St Paul, MN,
USA). Laboratory temperature was always within the range $17-24^{\circ} \mathrm{C}$. Data pertaining to the standing subject were subtracted from the steady state values, reached after about $4 \mathrm{~min}$ of moving, to obtain the net energy expenditure at each trial.

Metabolic work values in the present paper were expressed as the mechanical work in Joules per kilogram of body mass $(\mathrm{J} /(\mathrm{kg} \mathrm{m}))$, by assuming that $1 \mathrm{ml} \mathrm{O}_{2}=20.9$ $\mathrm{J}$ (for a mean respiratory quotient of about 0.95). The metabolic cost of locomotion $(\mathrm{C}, \mathrm{J} /(\mathrm{kg} \mathrm{m}))$ was calculated by subtracting the standing values from the measured energy expenditure and dividing by the speed. At each speed and gait, the apparent efficiency was calculated as the ratio between the mechanical and the metabolic work.

Statistical analysis was performed by using the unpaired $t$-test at each speed. Asterisks in the figures reflect two different levels of probability $\left({ }^{*} \mathrm{P}<0.05\right.$; $\left.{ }^{* *} \mathrm{P}<0.01\right)$.

\section{Results}

Wext (Fig. 1, upper panel) was not different between GHD patients and controls, with the exception of the
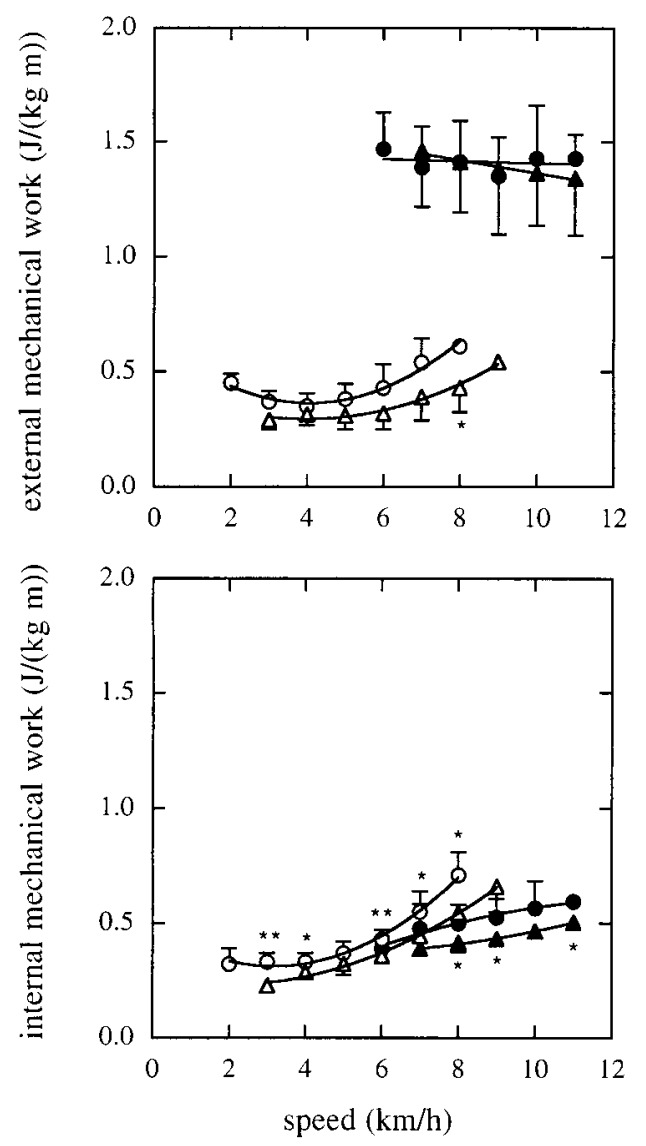

Figure 1 External mechanical work (upper panel) and internal mechanical work (lower panel) vs speed of walking/running. GHD patients (circles), controls (triangles); walking (open symbols), running (solid symbols). Results are means \pm s.D. ${ }^{*} P<0.05$; ${ }^{\star *} P<0.01$. 


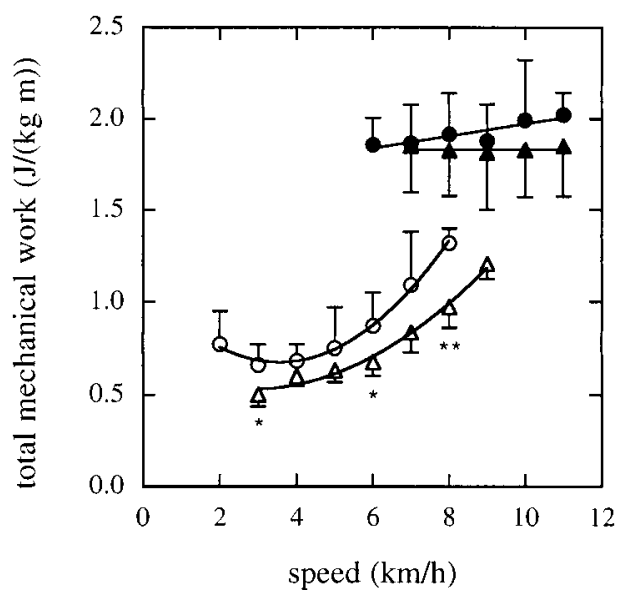

Figure 2 Total mechanical work vs speed. Symbols as in Fig. 1. ${ }^{*} P<0.05 ;{ }^{*} P<0.01$.

highest speed of walking, while Wint of patients (Fig. 1, lower panel) was significantly higher at almost all walking and running speeds. Wtot, the sum of Wint and Wext (Fig. 2), was higher in GHD patients than in controls, although the difference reached significance only at three speeds of walking.

Stride frequency was always significantly higher $(+11.2 \pm 2.6 \%$ for walking and $+11.3 \pm 2.1 \%$ for running) in GHD patients at all speeds of walking and running.

Metabolic data, collected in four GHD patients (nos 1-4), are shown in Fig. 3.

The average $C$ of walking was about the same in the two groups. However, the patients were unable to run at speeds higher than $8 \mathrm{~km} \mathrm{~h}^{-1}$ for the time needed to reach a metabolic steady state, thus only two speeds of running ( 7 and $8 \mathrm{~km} \mathrm{~h}^{-1}$ ) were actually comparable with those recorded in healthy controls.

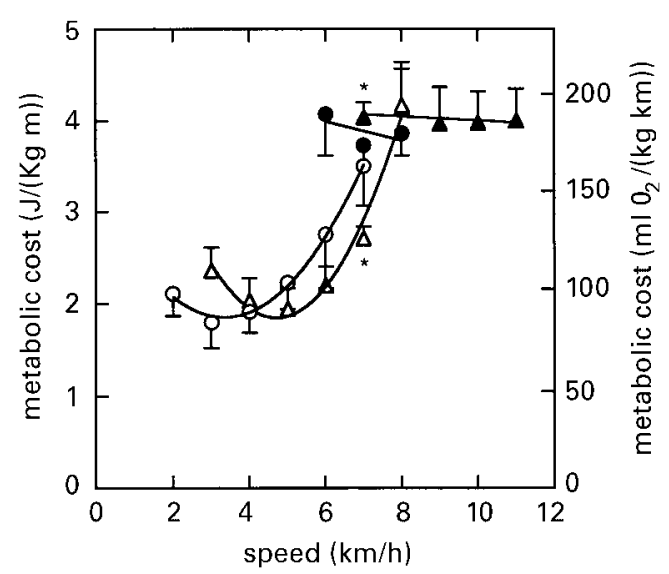

Figure 3 Mean values for metabolic cost vs speed of walking and running. Symbols as in Fig. 1. ${ }^{*} P<0.05$. The metabolic cost is expressed also in mechanical units, by considering $1 \mathrm{ml} \mathrm{O}_{2}=20.9 \mathrm{~J}$.

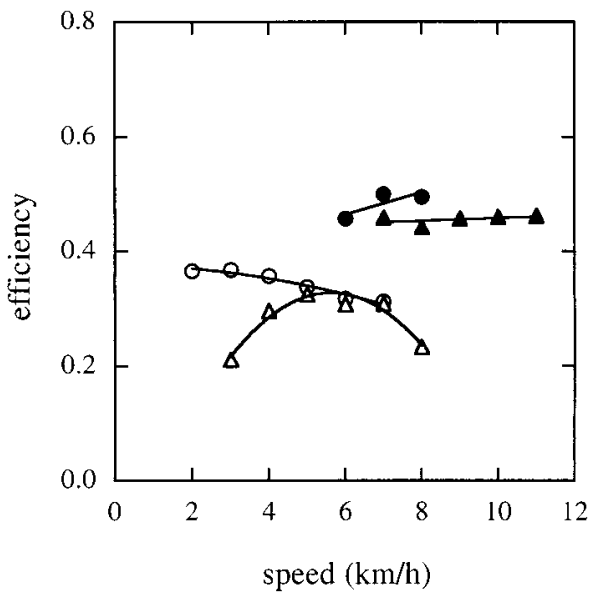

Figure 4 Apparent mechanical efficiency vs speed of walking and running. Symbols as in Fig. 1.

The apparent mechanical efficiency was somewhat higher in GHD patients at slow walking, while it was similar to controls in all the other conditions (see Fig. 4).

Figure 5 shows the recovery of mechanical energy, an index introduced by Cavagna et al. (11), which reflects the capacity of a moving body to save mechanical energy by means of the pendulum-like motion (an ideal pendulum moves with a percentage recovery of $100 \%$, i.e. it continuously exchanges the potential and the kinetic energy with no loss). The curves of percentage recovery were similar in shape in the two groups, although the curve for patients with GHD shifted towards lower speeds with respect to controls.

\section{Discussion}

The results reported in the present study show that short statured adults with childhood-onset GHD do not

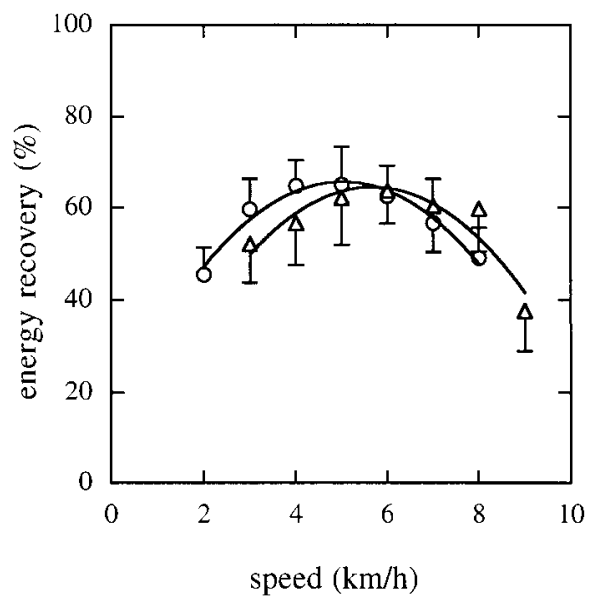

Figure 5 Energy recovery of walking in the two groups. Symbols as in Fig. 1. 
have a significant impairment of mechanical and metabolic profile of locomotion, differences from controls probably being due to their reduced body size.

Healthy controls were selected in an attempt to match their physical activity to that of patients with GHD; it is evident that our controls, matched for age, sex and exercise capacity, were not matched for body height. This limit in the interpretation of the results might have been overcome by selecting either taller patients with GHD or shorter controls (i.e. adults with extremely short stature, who however cannot be completely considered as 'normal' with respect to their GH secretion).

The mechanical external work was not significantly different between GHD patients and healthy controls, except for the highest speed of walking. On the other hand, the mechanical internal work was significantly higher at almost all speeds of walking (average: $+25.3 \%$ ) and running (average: $+22.0 \%$ ). According to papers on the determinants of the internal work $(12,13)$, part of the increase in GHD patients can be explained by the observed $+11.2 \%$ increase in the stride frequency (see below). The combined effect of internal and external work determined more evident differences in the mechanical total work between patients and controls, the differences being significant only at three speeds of walking.

The increase in stride frequency is in line with the pendulum paradigm referred to walking. In an ideal pendulum the passive oscillation frequency is related to the square root of the reciprocal of the shaft length. By applying this argument to humans, it can be demonstrated that the ratio between the stride frequency (f) of GHD and controls $(\mathrm{CON})$ is equal to:

$$
\frac{\mathrm{f}_{\mathrm{GHD}}}{\mathrm{f}_{\mathrm{CON}}}=\sqrt{\frac{\mathrm{l}_{\mathrm{CON}}}{\mathrm{l}_{\mathrm{GHD}}}}
$$

where $\mathrm{l}_{\mathrm{GHD}}$ and $\mathrm{l}_{\mathrm{CON}}$ refer to the leg length of the two subject groups.

The first term of the equation is equal to 1.112 , as shown in the Results, and the second one was calculated from the average leg length $(0.76$ and $0.92 \mathrm{~m}$ for GHD and control subjects respectively) as 1.102. The agreement between the two values indicates that the pendulum paradigm is operating equally in the two groups. Moreover, since the observed frequency ratio is lower than the 'straight' ratio between leg lengths $(=1.21)$, the stride length has to be greater (relative to their body size) in patients. However, to explain the increase in the mechanical internal work of walking $(+25 \%)$, we have to hypothesize that the duty factor, i.e. the fraction of the stride cycle at which one foot is on the ground, is also increased in GHD patients. While not directly investigated in this paper, the duty factor has recently been shown to be one of the main determinants of the internal work (13).

The relationship between metabolic cost of locomotion and speed, shown in Fig. 3, reveals a shift towards slow velocities of the walking curve in GHD patients. The optimum walking speed, i.e. the speed at which the unit distance is travelled with the least metabolic consumption, is lower $\left(3.5 \mathrm{~km} \mathrm{~h}^{-1}\right)$ in GHD patients than in controls $\left(5.0 \mathrm{~km} \mathrm{~h}^{-1}\right)$. Such a shift could be partly explained by the exchange between potential and kinetic energy of the body centre of mass (see below). No notable differences between the two groups occurred in the metabolic cost of running.

As a result, from a higher total mechanical work and almost the same metabolic cost, the efficiency of walking in GHD patients was slightly higher than in controls, mainly at the slowest speeds (see Fig. 4). The same leftshift observed in the metabolic curve could have been hidden inefficiency by the data variability of single components of the mechanical work and metabolic cost.

The percentage energy recovery is maximal at about $6 \mathrm{~km} \mathrm{~h}^{-1}$ for the control subjects, while for GHD patients the maximum occurs at a lower speed $(5 \mathrm{~km}$ $\mathrm{h}^{-1}$ ). However, the pattern of recovery seems similar in the two groups, being shifted to the left side in patients. This finding is the consequence of the difference in size between patients and controls, as previously suggested by Cavagna et al. (14). One way to compare the motion of differently sized bodies is to replace the speed units on the graph abscissa with a 'dimensionless' speed, which takes into account the dynamic similarity of the bodies. Differently from comparisons based on geometry, the theory of dynamic similarity also preserves the forces at different scales, particularly those involved in the transformation of potential into kinetic energy, and vice versa. For this goal a dimensionless speed, known as the Froude number and equal to:

$$
\mathrm{Fr}=\frac{\mathrm{s}^{2}}{\mathrm{gl}}
$$

has been designed (it can be shown that it is proportional to the ratio between kinetic and potential energies). In the equation, $\mathrm{s}$ is the progression speed, $\mathrm{g}$ is gravity acceleration $\left(9.8 \mathrm{~m} / \mathrm{s}^{2}\right)$ and $\mathrm{l}$ is a characteristic body length (e.g. the leg length). Two differently sized pendulums move in a dynamically similar fashion when the respective speeds correspond to the same Froude number.

Both metabolic cost and the mechanical energy recovery have been plotted versus the Froude number in Fig. 6 by using, for each subject group, the proper average leg length. The dynamic similarity between GHD patients and control subjects is witnessed by the tendency to overimpose of the two curves in each graph. Particularly, the recovery values almost superimpose, confirming the pendulum-like paradigm of walking at corresponding speeds in patients.

On the metabolic side, GHD patients exhibited a greater fatiguability than healthy controls, since they were significantly less economical at the higher speeds of walking and they were unable to run faster than $8 \mathrm{~km}$ $\mathrm{h}^{-1}$. These results could be somewhat in contrast with 

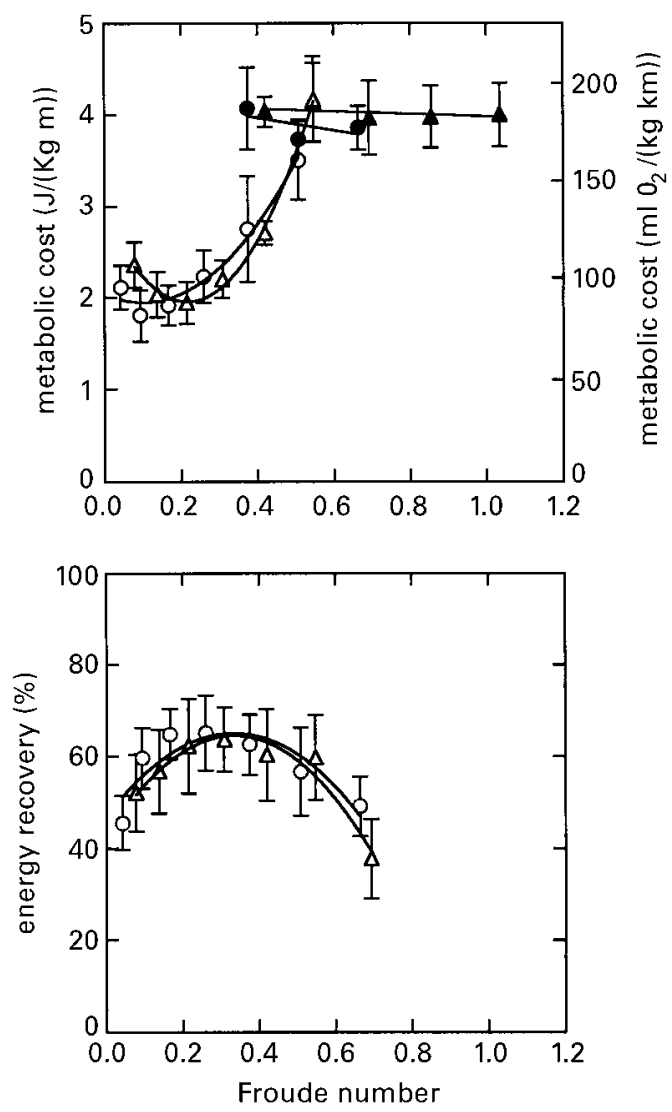

Figure 6 Metabolic energy cost of locomotion (upper panel) and energy recovery (lower panel) as a function of the dimensionless speed known as the Froude number (see text for details). Symbols as in Fig. 1. The metabolic cost is expressed also in mechanical units, by considering $1 \mathrm{ml} \mathrm{O}_{2}=20.9 \mathrm{~J}$.

the findings that both muscle strength 'normalized' for CSA $(3,5)$ and fibre type distribution of these patients were similar to those found in controls (7).

A plausible explanation could be the scarce habituation to treadmill locomotion of these patients, although it has been shown that the cost of running remains constant after the 7 th trial and is only $3 \%$ higher than the final value at the 3rd trial (15). Furthermore, the greater fatiguability observed in GHD patients than in controls could be due to the impairment of cardiac structure and function, as previously demonstrated (16). It is also possible that the present group of dwarfs was composed of rather sedentary individuals with a low maximum aerobic power. Their average heart rate

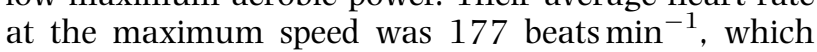
from their measured resting and age-estimated maximum heart rate would correspond to about $90 \%$ of the maximum, so that they had to rely, in part, on anaerobic metabolism.

At the two lowest speeds of walking, GHD patients showed a lower cost than controls. This could be due to the fact that the specific energy expenditure at rest is higher for lighter (GHD) than for heavier (controls) individuals, as predicted by allometric rules. At the lowest values of energy expenditure, such as those measured at slow walking, resting energy expenditure represents a significant fraction of the overall energy expenditure, affecting the calculation of cost.

The cost of running was also significantly lower in GHD patients (about $7-8 \%$ ), at least for the two matching speeds, a surprising result indeed as cost was found to be negatively related both to body mass and to height (17). It is interesting that the same differences have been found between Pygmies $(153 \mathrm{~cm})$ and healthy controls (18).

Further additional data (particularly at higher speeds) are needed to confirm these results, which point to the possibility that GHD patients are able to store more elastic energy, perhaps due to having tendons disproportionally thinner than normal subjects.

Although in our experiments the spontaneous transition from walking to running was not specifically looked at, it appears that GHD patients changed gait at a lower speed than controls, as is commonly observed in children. Furthermore, the optimal speed of walking, the speed at which cost and external work are minimal, is lower for GHD patients (about $3.5 \mathrm{~km} \mathrm{~h}^{-1}$ ) than for healthy controls (about $5 \mathrm{~km} \mathrm{~h}^{-1}$ ), being very similar to that recorded in children of the same stature (14). The optimal speed does not correspond to the speed at which percentage recovery is maximal, the latter depending only on external work, the former depending also on internal work and on the muscular efficiency at different velocities of contraction.

In conclusion, it appears that both the mechanics and energetics of locomotion in short-statured adults with childhood-onset GHD are not strikingly different from those of healthy controls, in agreement with our previous observations (3, 5, 7) demonstrating a substantial 'normality' in this group of GHD patients.

Despite the small sample size, the results from the present study are consistent with previous data on control subjects and with data predicted from the principle of dynamic similarity for smaller subjects (19).

Although the findings of harmonic and co-ordinated allometric transformations in these patients seem to hypothesize that GH substitution therapy is not necessary to maintain normal muscle mass and function, the possibility of a different metabolic role of $\mathrm{GH}$ in normal statured adults with childhood-onset GHD and in those with acquired GHD cannot be ruled out, taking into account the well-recognized heterogeneity of the adult GHD syndrome (20).

\section{Acknowledgements}

This work was partially supported by Progetti di Ricerca Corrente, Istituto Auxologico Italiano, IRCCS, Milano. This study would not have been possible without the support of the medical staff at the Research Center for Growth Disorders, Italian Institute for Auxology, Milan. 
The authors are very grateful to all the patients who participated enthusiastically and collaboratively in our research.

\section{References}

1 Cuneo C, Salomon F, Wiles CM \& Sonksen PH. Skeletal muscle performance in adults with growth hormone deficiency. Hormone Research 19904 (Suppl) 55-60.

2 Cuneo C, Salomon F, Wiles CM, Hesp R \& Sonksen PH. Growth hormone treatment in growth hormone-deficient adults. I. Effects on muscle mass and strength. Journal of Applied Physiology 1991 70 688-694.

3 Sartorio A \& Narici M. Growth hormone (GH) treatment in GHdeficient adults: effects on muscle size, strength and neura activation. Clinical Physiology 199414 527-537.

4 Rutherford M, Beshyah SA, Schott J, Watkins Y \& Johnston DG. Contractile properties of the quadriceps muscle in growth hormone-deficient hypopituitary adults. Clinical Science 199588 67-71.

5 Sartorio A, Narici M, Conti A, Monzani M \& Faglia G. Quadriceps and hand-grip strength in adults with childhood-onset growth hormone deficiency. European Journal of Endocrinology 1995132 $37-41$

6 Johansson G, Grimby G, Stibrant Sunnerhagen K \& Bengtsson B. Two years of growth hormone $(\mathrm{GH})$ treatment increase isometric and isokinetic muscle strength in GH-deficient adults. Journal of Clinical Endocrinology and Metabolism 199782 2877-2884.

7 Bottinelli R, Narici M, Pellegrino MA, Kayser B, Canepari M, Faglia $\mathrm{G}$ et al. Contractile properties and fibre type distribution of quadriceps muscles in adults with childhood-onset growth hormone deficiency. Journal of Clinical Endocrinology and Metabolism 199782 4133-4138.

8 Dempster WT, Gabel WC \& Felts WJL. The anthropometry of manual work space for the seated subject. American Journal of Physical Anthropology 195917 289-317.

9 Cavagna GA \& Kaneko M. Mechanical work and efficiency in level walking and running. Journal of Physiology 1977268 467-481.
10 D'Amico M \& Ferrigno G. Technique for the evaluation of derivatives from noisy biomechanical displacement data using a model based bandwidth-selection procedure. Medical and Biological Engineering and Computing 199028 407-415.

11 Cavagna GA, Thys H \& Zamboni A. The sources of external work in level walking and running. Journal of Physiology 1976262 639-657.

12 Minetti AE \& Saibene F. Mechanical work rate minimization and freely chosen stride frequency of human walking: a mathematical model. Journal of Experimental Biology 1992170 19-34.

13 Minetti AE. A model equation for the prediction of mechanical internal work of terrestrial locomotion. Journal of Biomechanics $199831463-468$.

14 Cavagna GA, Franzetti P \& Fuchimoto T. The mechanics of walking in children. Journal of Physiology 1983343 323-339.

15 Brueckner JC, Atchou G, Capelli C, Duvallet A, Barrault D, Jousselin E et al. The energy cost of running increases with the distance covered. European Journal of Applied Physiology 199162 385-389.

16 Sartorio A, Ferrero S, Conti A, Bragato R, Malfatto G \& Leonetti G. Adults with childhood-onset growth hormone deficiency: effects of growth hormone treatment on cardiac structure. Journal of Internal Medicine 1997241 515-520.

17 Bourdin M, Pastene J, Germaine M \& Lacour JR. Influence of training, sex, age and body mass on the energy cost of running. European Journal of Applied Physiology 199366 439-444.

18 Minetti AE, Saibene F, Ardigò LP, Atchou G, Schena F \& Ferretti G. Pygmy locomotion. European Journal of Applied Physiology 1994 68 285-290.

19 McNeill Alexander R. Optimization and gaits in the locomotion of vertebrates. Physiological Review 198969 1199-1227.

20 Attanasio AF, Lamberts SWJ, Matranga AMC, Birkett MA, Bates $\mathrm{PC}$, Valk NK et al. Adult growth hormone (GH)-deficient patients demonstrate heterogeneity between childhood onset and adult onset before and during human GH treatment. Journal of Clinical Endocrinology and Metabolism 199782 82-88.

Received 15 March 1999

Accepted 24 August 1999 